\title{
Quantization Design for Unconstrained Distributed Optimization
}

\author{
Ye Pu, Melanie N. Zeilinger and Colin N. Jones
}

\begin{abstract}
We consider an unconstrained distributed optimization problem and assume that the bit rate of the communication in the network is limited. We propose a distributed optimization algorithm with an iteratively refining quantization design, which bounds the quantization errors and ensures convergence to the global optimum. We present conditions on the bit rate and the initial quantization intervals for convergence, and show that as the bit rate increases, the corresponding minimum initial quantization intervals decrease. We prove that after imposing the quantization scheme, the algorithm still provides a linear convergence rate, and furthermore derive an upper bound on the number of iterations to achieve a given accuracy. Finally, we demonstrate the performance of the proposed algorithm and the theoretical findings for solving a randomly generated example of a distributed least squares problem.
\end{abstract}

\section{INTRODUCTION}

Distributed optimization methods for networked systems that have many coupled sub-systems and must act based on local information, are critical in many engineering problems, e.g. resource allocation, distributed estimation and distributed control problems. The algorithms are required to solve a global optimization problem in a distributed fashion subject to communication constraints.

Inexact distributed optimization methods are attracting increasing attention since, in practice, these techniques have to deal with both the inexact solution of local problems as well as noise caused by unreliable or limited communication, e.g., transmission failures and quantization errors. The question is then how these errors affect the algorithm and under which conditions convergence can still be guaranteed. Inexact optimization algorithms aim at answering these questions. Recent works include [4] and [8]. In [4], the authors propose an inexact decomposition algorithm for solving distributed optimization problems by employing smoothing techniques and an excessive gap condition. In [8], an inexact splitting method, named the inexact fast alternating minimization algorithm, is proposed and applied to distributed optimal control problems. Some other related references for inexact optimization algorithms include [3], [6] and [9]. In [9], an

Y. Pu and C.N. Jones are with the Automatic Control Lab, École Polytechnique Fédérale de Lausanne, EPFL-STI-IGM-LA Station 9 CH-1015 Lausanne, Switzerland, e-mail: $\{y \cdot p u, c o l i n . j o n e s\}$ depfl.ch.

M.N. Zeilinger is with the Empirical Inference Department, Max Planck Institute for Intelligent Systems, 72076 Tübingen, Germany, e-mail: melanie.zeilinger@tuebingen.mpg.de.

This work has received funding from the European Research Council under the European Union's Seventh Framework Programme (FP/20072013)/ ERC Grant Agreement n. 307608. The research of M. N. Zeilinger has received funding from the EU FP7 under grant agreement no. PIOFGA-2011-301436-“COGENT". inexact proximal-gradient method, as well as its accelerated version, are introduced. The proximal gradient method, also known as the iterative shrinkage-thresholding algorithm (ISTA) [1], has two main steps: the first step is to compute the gradient of the smooth objective and the second step is to solve the proximal minimization. The conceptual idea of the inexact proximal-gradient method is to allow errors in these two steps, i.e. an error in the calculation of the gradient and an error in the proximal minimization. The results in [9] show convergence properties of the inexact proximalgradient method and provide conditions on the errors, under which convergence of the algorithm can be guaranteed. In this paper, we focus on communication errors and build on the results of [9].

We consider an unconstrained distributed optimization problem, where each sub-system has a local cost function, that involves both local and neighbouring variables. The problem is solved in a distributed manner with only local communication. In many problems, the communication bandwidth is limited requiring quantization of the information exchanged among the neighbouring sub-systems. The quantization process results in inexact iterations throughout the distributed optimization algorithm, which affect its convergence. Related work includes [2], [5], [10] and [7], which study the effects of quantization on the performance of averaging or distributed optimization algorithms.

We propose a distributed optimization algorithm with progressive quantization design building on the work in [9] and [10]. The main idea behind the proposed method is to apply the inexact gradient method to the distributed optimization problem and to employ the error conditions, which guarantee convergence to the global optimum, to design a progressive quantizer. Motivated by the linear convergence upper-bound of the optimization algorithm, the range of the quantizer is set to reduce linearly at a rate smaller than one and larger than the rate of the algorithm, in order to refine the information exchanged in the network with each iteration and achieve overall converge to the global optimum. The proposed quantization method is computationally cheap and consistent throughout the iterations as every node implements the same quantization procedure. The detailed contributions of this work are the following:

- We propose an unconstrained distributed optimization algorithm with iteratively refining quantization design. This method computes an optimal solution of a distributed optimization problem with locally coupled cost function under limited communication, i.e. each subsystem can only communicate with its neighbours, and at each iteration, only a fixed number of bits can be 
transmitted.

- We present conditions on the parameters of the quantizers, i.e. the number of bits and the initial quantization intervals, which guarantee convergence of the algorithm.

- We prove that the algorithm with the quantization design preserves the linear convergence rate, and furthermore derive an upper bound on the number of iterations to achieve a given accuracy.

- We demonstrate the performance of the proposed method and the theoretical results for solving a randomly generated example of a least squares problem with 20 subsystems.

\section{PRELIMINARIES}

\section{A. Notation}

Let $v \in \mathbb{R}^{n_{v}}$ be a vector. $\|v\|$ and $\|v\|_{\infty}$ denote the $l_{2}$ and infinity norms of $v$, respectively. Note that $\|v\|_{\infty} \leq\|v\|_{2} \leq$ $\sqrt{n_{v}}\|v\|_{\infty}$. Let $f: \Theta \rightarrow \Omega$ be a strongly convex function; $\sigma_{f}$ denotes the convexity modulus $\langle p-q, x-y\rangle \geq \sigma_{f}\|x-y\|^{2}$, where $p \in \partial f(x), q \in \partial f(y)$ and $\partial f(\cdot)$ denotes the set of sub-gradients of the function $f$ at a given point. $L(f)$ denotes a Lipschitz constant of the function $f$, i.e. $\left\|f\left(x_{1}\right)-f\left(x_{2}\right)\right\| \leq$ $L(f)\left\|x_{1}-x_{2}\right\|, \forall x_{1}, x_{2} \in \Theta$.

\section{B. Inexact gradient method}

In this section, an inexact gradient method, which is a special case of the inexact proximal-gradient method proposed in [9], will be introduced. The convergence theorem based on the theoretical results in [9] will be presented. Inexact gradient method addresses optimization problems of the form in Problem II.1 and requires Assumption II.2. The inexact gradient method is given in Algorithm 1.

\section{Problem II.1.}

$$
\min _{w \in \mathbb{R}^{n_{w}}} \phi(w) .
$$

Assumption II.2. $\phi$ is a strongly convex function with a convexity modulus $\sigma_{\phi}$ and Lipschitz continuous gradient with constant $L(\nabla \phi)$.

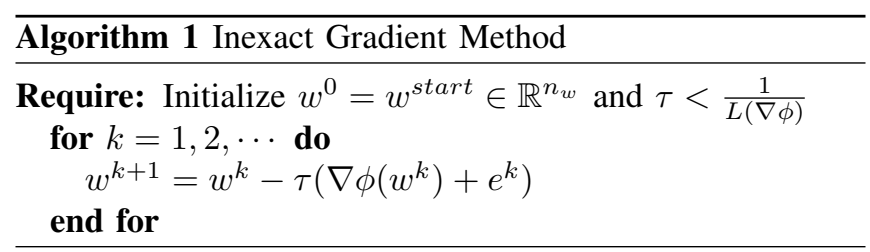

The inexact proximal-gradient method proposed in [9] considers two kinds of errors: an error in the gradient calculation and an error in the computation of the proximal minimization. In Algorithm 1, we only focus on the first kind of error and denote it by $e^{k}$ at each iteration $k$. The following proposition states the convergence property of inexact gradient method, which can be easily derived from Proposition 4 in [9], by considering the second objective and the error in the computation of the proximal minimization to be zero.
Proposition II.3 (From Proposition 4 in [9]). Let $\left\{w^{k}\right\}$ be generated by inexact gradient method defined in Algorithm 1. If Assumption II.2 holds, then for any $k \geq 1$ we have

$$
\left\|w^{k}-w^{\star}\right\| \leq(1-\gamma)^{k}\left(\left\|w^{0}-w^{\star}\right\|+\Gamma^{k}\right),
$$

where $\gamma=\frac{\sigma_{\phi}}{L(\nabla \phi)}$ and $w^{0}$ and $w^{\star}$ denote the starting sequence of Algorithm 1 and the optimal solution of Problem II.1, respectively, and

$$
\Gamma^{k}=\sum_{p=1}^{k}(1-\gamma)^{-p} \cdot \frac{\left\|e^{p}\right\|}{L(\nabla \phi)}
$$

As discussed in [9], the upper-bound in Proposition II.3 allows one to derive sufficient conditions on the error sequence $\left\{e^{k}\right\}$ for the convergence of the algorithm to the optimal solution $w^{*}$ :

- If the series $\left\{\left\|e^{k}\right\|\right\}$ decreases linearly at a rate of $\kappa<$ $(1-\gamma)$, then $\left\|w^{k}-w^{\star}\right\|$ converges linearly with the rate $1-\gamma$.

- If the series $\left\{\left\|e^{k}\right\|\right\}$ decreases linearly at a rate of $(1-$ $\gamma)<\kappa<1$, then $\left\|w^{k}-w^{\star}\right\|$ converges linearly with the rate $\kappa$.

- If the series $\left\{\left\|e^{k}\right\|\right\}$ decreases linearly at a rate of $\kappa=$ $(1-\gamma)$, then $\left\|w^{k}-w^{\star}\right\|$ converges linearly at a rate of $O\left(k(1-\gamma)^{k}\right)$.

\section{Uniform quantizer}

Let $x$ be a real number. A uniform quantizer with a quantization step-size $\Delta$ and the mid-value $\bar{x}$ can be expressed as

$$
Q(x)=\bar{x}+\operatorname{sgn}(x-\bar{x}) \cdot \Delta \cdot\left\lfloor\frac{\|x-\bar{x}\|}{\Delta}+\frac{1}{2}\right\rfloor,
$$

where $\operatorname{sgn}(\cdot)$ is the sign function. The parameter $\Delta$ is equal to $\Delta=\frac{l}{2^{n}}$, where $l$ represents the size of the quantization interval and $n$ is the number of bits sent by the quantizer. In this paper, we assume that $n$ is a fixed number, which means that the quantization interval is set to be $\left[\bar{x}-\frac{l}{2}, \bar{x}+\frac{l}{2}\right]$. The quantization error is upper-bounded by

$$
x-Q(x) \leq \frac{\Delta}{2}=\frac{l}{2^{n+1}} .
$$

For the case that the input of the quantizer and the midvalue are not real numbers, but vectors of dimension $n_{x}$, the quantizer $Q$ is composed of $n_{x}$ independent scalar quantizers in (2) with the same quantization interval $l$ and corresponding mid-value. In this paper, we design a uniform quantizer denoted as $Q^{k}(\cdot)$ with changing quantization interval $l^{k}$ and mid-value $\bar{x}^{k}$ at every iteration $k$ of the optimization algorithm.

\section{Distributed OPTIMIZATION WITH LIMITED COMMUNICATION}

In this section, we will apply the inexact gradient method to an an unconstrained distributed optimization problem, where the error results from limited local communication. We assume that at each iteration, only a fixed number 
of bits can be transmitted and the exchanged information must be quantized. We design a uniform quantizer using a varying quantization interval and mid-value to refine the exchanged information at each iteration. Motivated by the linear complexity bound of the inexact gradient method in Theorem II.3, the quantization interval is updated by a linearly decreasing function, and as a result the induced quantization noise decreases at the same rate. We show conditions on the number of bits, the initial quantization intervals and the linear decrease rate, under which the inexact distributed algorithm converges to the global optimum and the linear convergence rate is preserved.

We consider a network of $M$ agents. The agents interact and communicate according to a fixed undirected graph $G=$ $(\mathcal{V}, \mathcal{E})$. The vertex set $\mathcal{V}=\{1,2, \cdots, M\}$ represents the agents and the edge set $\mathcal{E} \subseteq \mathcal{V} \times \mathcal{V}$ specifies pairs of agents that can communicate. If $(i, j) \in \mathcal{E}$, we say that agents $i$ and $j$ are neighbours, and we denote by $\mathcal{N}_{i}=\{j \mid(i, j) \in \mathcal{E}\}$ the set of the neighbours of agent $i$. Note that $\mathcal{N}_{i}$ includes $i$. We denote $d$ as the degree of $G$. The state of agent $i$ and the global state are denoted by $x_{i}$ and $x=\left[x_{1}^{T}, \cdots, x_{M}^{T}\right]^{T}$, respectively. The dimension of the local state $x_{i}$ is denoted by $m_{i}$ and the maximum dimension of the local states is denoted by $\bar{m}$, i.e. $\bar{m}:=\max _{1 \leq i \leq M} m_{i}$. The concatenation of the state of agent $i$ and the states of its neighbours is denoted by $x_{\mathcal{N}_{i}}$. With the selecting matrices $E_{i}$ and $F_{i}$, they can be represented as $x_{\mathcal{N}_{i}}=E_{i} x$ and $x_{i}=F_{i} x_{\mathcal{N}_{i}}$. Note that $\left\|E_{i}\right\|=\left\|F_{i}\right\|=1$. We solve a distributed optimization problem of the form in Problem III.1:

\section{Problem III.1.}

$$
\min _{x \in \mathbb{R}^{n_{x}}} f(x)=\sum_{i=1}^{M} f_{i}\left(x_{\mathcal{N}_{i}}\right) .
$$

Assumption III.2. We assume that the global cost function $f(\cdot)$ is strongly convex with a convexity modulus $\sigma_{f}$

$$
\left(\nabla f\left(x_{1}\right)-\nabla f\left(x_{2}\right)\right)^{T}\left(x_{1}-x_{2}\right) \geq \sigma_{f}\left\|x_{1}-x_{2}\right\|^{2}
$$

and Lipschitz continuous gradient with constant $L$

$$
\left\|\nabla f\left(x_{1}\right)-\nabla f\left(x_{2}\right)\right\| \leq L\left\|x_{1}-x_{2}\right\| .
$$

Assumption III.3. We assume that every local cost function has Lipschitz continuous gradient with constant $L_{i}$, and denote $L_{\max }$ as the maximum Lipschitz constant of the local functions, i.e. $L_{\max }:=\max _{1 \leq i \leq M} L_{i}$.

We apply the gradient method to Problem III.1. The parameter $\gamma$ is equal to

$$
\gamma=\frac{\sigma_{f}}{L} .
$$

Algorithm 2 presents the proposed distributed algorithm with progressive quantization design, in which each agent updates its state, computes the gradient of its cost function locally and sends the updated value to its neighbours. At each iteration, each agent sends information to its neighbours twice: the updated state $x_{i}^{k}$, and the gradient $\nabla f_{i}^{k}$. Since at each iteration only a fixed number of bits can be sent, we design two uniform quantizers $Q_{\alpha, i}^{k}$ and $Q_{\beta, i}^{k}$ for every agent $i$ using the formulation introduced in Section II-C with a fixed number of bits $n$, changing quantization intervals $l_{\alpha, i}^{k}$ and $l_{\beta, i}^{k}$ and changing mid-values $\bar{x}_{\alpha, i}^{k}$ and $\bar{\nabla} f_{\beta, i}^{k}$ for transmitting $x_{i}^{k}$, and $\nabla f_{i}^{k}$ at every iteration $k$. The quantized values are denoted by $\hat{x}_{i}^{k}$ and $\hat{\nabla} f_{i}^{k}$, and the quantization errors are denoted by $\alpha_{i}^{k}=\hat{x}_{i}^{k}-x_{i}^{k}$ and $\beta_{i}^{k}=\hat{\nabla} f_{i}^{k}-$ $\nabla f_{i}^{k}$. In the following, we present three lemmas to link

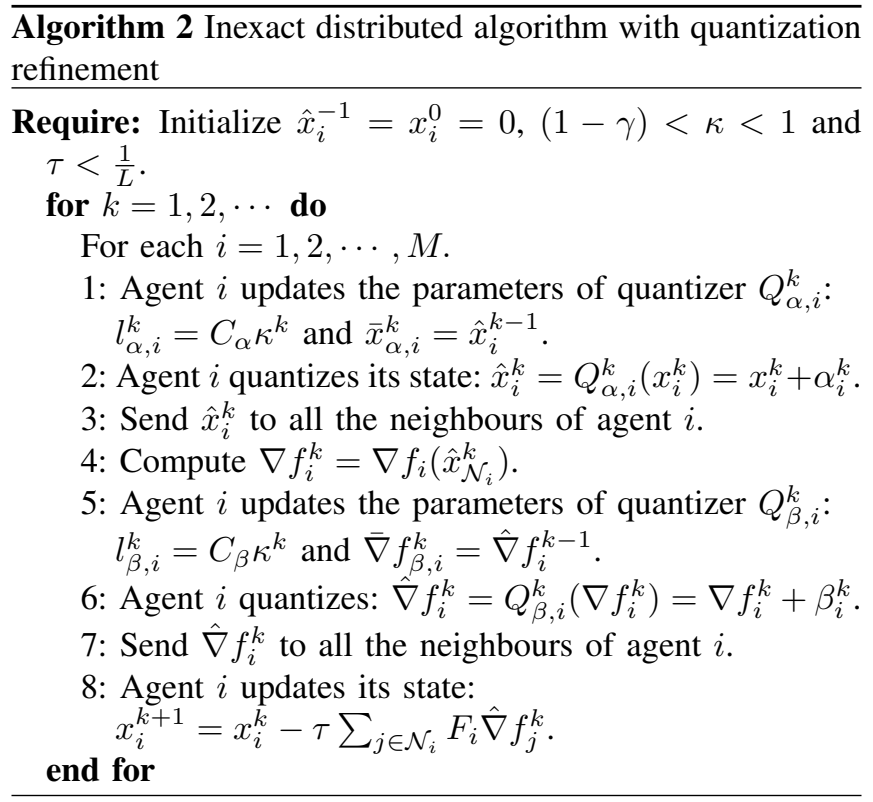

Algorithm 2 to the inexact gradient method and to prove that Algorithm 2 converges linearly to the global optimum despite the quantization errors. Lemma III.4 shows that the inexactness resulting from quantization in Algorithm 2 is equivalent to the error of the computation of the gradient in Algorithm 1. Lemma III.5 states that if at each iteration the values $x_{i}^{k}$ and $\nabla f_{i}^{k}$ fall inside the quantization intervals, then the errors caused by quantization decrease linearly and the algorithm converges to the global optimum at the same rate. Lemma III.9 gives conditions on the number of bits and the initial quantization intervals, which guarantee that $x_{i}^{k}$ and $\nabla f_{i}^{k}$ fall inside the quantization intervals for each iteration. Once we prove the three lemmas, we are ready to present the main theorem.

Lemma III.4. Algorithm 2 is equivalent to applying the inexact gradient method in Algorithm 1 to Problem III.1 with $\phi=f$ and

$e^{k}=\sum_{i=1}^{M} E_{i}^{T} \nabla f_{i}\left(\hat{x}_{\mathcal{N}_{i}}^{k-1}\right)+\sum_{i=1}^{M} E_{i}^{T} \beta_{i}^{k}-\sum_{i=1}^{M} E_{i}^{T} \nabla f_{i}\left(x_{\mathcal{N}_{i}}^{k-1}\right)$.

Furthermore, $\left\|e^{k}\right\|$ is upper-bounded by

$$
\left\|e^{k}\right\| \leq \sum_{i=1}^{M} L_{i} \cdot \sum_{j \in \mathcal{N}_{i}}\left\|\alpha_{j}^{k}\right\|+\sum_{i=1}^{M}\left\|\beta_{i}^{k}\right\| .
$$


Proof: The gradient computation error $e^{k}$ in Algorithm 1 is equal to

$$
\begin{aligned}
e^{k} & =\hat{\nabla} f\left(\hat{x}^{k-1}\right)-\nabla f\left(x^{k-1}\right) \\
& =\sum_{i=1}^{M} E_{i}^{T} \hat{\nabla} f_{i}\left(\hat{x}_{\mathcal{N}_{i}}^{k-1}\right)-\sum_{i=1}^{M} E_{i}^{T} \nabla f_{i}\left(x_{\mathcal{N}_{i}}^{k-1}\right) \\
& =\sum_{i=1}^{M} E_{i}^{T} \nabla f_{i}\left(\hat{x}_{\mathcal{N}_{i}}^{k-1}\right)+\sum_{i=1}^{M} E_{i}^{T} \beta_{i}^{k}-\sum_{i=1}^{M} E_{i}^{T} \nabla f_{i}\left(x_{\mathcal{N}_{i}}^{k-1}\right) .
\end{aligned}
$$

Then,

$$
\begin{aligned}
\left\|e^{k}\right\| & \leq \sum_{i=1}^{M}\left\|E_{i}^{T}\right\| \cdot L_{i} \cdot\left\|\hat{x}_{\mathcal{N}_{i}}^{k-1}-x_{\mathcal{N}_{i}}^{k-1}\right\|+\sum_{i=1}^{M}\left\|\beta_{i}^{k}\right\| \\
& \leq \sum_{i=1}^{M} L_{i} \cdot \sum_{j \in \mathcal{N}_{i}}\left\|\alpha_{j}^{k}\right\|+\sum_{i=1}^{M}\left\|\beta_{i}^{k}\right\| .
\end{aligned}
$$

From the discussion in Section II-B, we know that if $\left\|e^{k}\right\|$ decreases linearly at a rate larger than $(1-\gamma)$, then $\left\|w^{k}-w^{\star}\right\|$ converges linearly at the same rate as $\left\|e^{k}\right\|$. Lemma III.5 brings Algorithm 2 close to this goal. It shows that if the values of $x_{i}^{k}$ and $\nabla f_{i}^{k}$ always fall inside the quantization interval, then the computational error of the gradient $e^{k}$ as well as $\left\|w^{k}-w^{\star}\right\|$ decrease linearly with the constant $\kappa$.

Lemma III.5. Given $k$, if for all $1 \leq p \leq k$ the values of $x_{i}^{p}$ and $\nabla f_{i}^{p}$ fall inside of the quantization intervals of $Q_{\alpha, i}^{p}$ and $Q_{\beta, i}^{p}$, i.e. $\left\|x_{i}^{k}-\bar{x}_{\alpha, i}^{k}\right\|_{\infty} \leq \frac{l_{\alpha, i}^{k}}{2}$ and $\left\|\nabla f_{i}^{k}-\bar{\nabla} f_{\beta, i}^{k}\right\|_{\infty} \leq \frac{l_{\beta, i}^{k}}{2}$, then the sequence $\left\|e^{p}\right\|$ decreases linearly at the rate of $\kappa$

$$
\left\|e^{p}\right\| \leq C \kappa^{p}
$$

where $C=\frac{M d m\left(L_{\max } C_{\alpha}+C_{\beta}\right)}{2^{n+1}}$, and $\left\|x^{p+1}-x^{\star}\right\|$ converges linearly at the rate $\kappa$ and satisfies

$$
\left\|x^{p+1}-x^{\star}\right\| \leq \kappa^{p+1}\left[\left\|x^{0}-x^{\star}\right\|+\frac{C \kappa}{L(\kappa+\gamma-1)}\right] .
$$

Proof: From the property of the uniform quantizer, we know that if $x_{i}^{p}$ and $\nabla f_{i}^{p}$ fall inside of the quantization intervals of $Q_{\alpha, i}^{p}$ and $Q_{\beta, i}^{p}$, then the quantization errors $\alpha_{i}^{p}$ and $\beta_{i}^{p}$ are upper-bounded by

$$
\begin{aligned}
& \left\|\alpha_{i}^{p}\right\| \leq m_{i} \cdot \frac{l_{\alpha, i}^{p}}{2^{n+1}} \leq \bar{m} \cdot \frac{l_{\alpha, i}^{p}}{2^{n+1}} \\
& \left\|\beta_{i}^{p}\right\| \leq \sum_{j \in \mathcal{N}_{i}} m_{j} \cdot \frac{l_{\beta, i}^{p}}{2^{n+1}} \leq d \cdot \bar{m} \cdot \frac{l_{\beta, i}^{p}}{2^{n+1}} .
\end{aligned}
$$

From Lemma III.4, we have

$$
\left\|e^{p}\right\| \leq \sum_{i=1}^{M} L_{i} \cdot \sum_{j \in \mathcal{N}_{i}} \frac{\bar{m} l_{\alpha, j}^{p}}{2^{n+1}}+\sum_{i=1}^{M} \frac{d \bar{m} l_{\beta, i}^{p}}{2^{n+1}} .
$$

Since the quantization intervals are set to $l_{\alpha, j}^{p}=C_{\alpha} \kappa^{p}$ and $l_{\beta, j}^{p}=C_{\beta} \kappa^{p}$, it implies that

$$
\left\|e^{p}\right\| \leq \frac{M L_{\max } d \bar{m} C_{\alpha} \kappa^{p}}{2^{n+1}}+\frac{M d \bar{m} C_{\beta} \kappa^{p}}{2^{n+1}}=C \kappa^{p},
$$

with $C=\frac{M d \bar{m}\left(L_{\max } C_{\alpha}+C_{\beta}\right)}{2^{n+1}}$. Proposition II.3 and $(1-\gamma)<$ $\kappa$ imply that for $1 \leq p \leq k$

$$
\begin{aligned}
\left\|x^{p+1}-x^{\star}\right\| & \leq(1-\gamma)^{p+1}\left\|x^{0}-x^{\star}\right\| \\
& +\frac{C}{L} \sum_{q=1}^{p+1} \kappa^{q}(1-\gamma)^{p+1-q} \\
& \leq \kappa^{p+1}\left[\left\|x^{0}-x^{\star}\right\|+\frac{C}{L} \sum_{q=1}^{p+1} \frac{(1-\gamma)^{p+1-q}}{\kappa^{p+1-q}}\right] .
\end{aligned}
$$

Since $0<(1-\gamma)<\kappa<1$, by using the property of geometric series, we get that the expression above is equal to

$$
\begin{aligned}
& =\kappa^{p+1}\left[\left\|x^{0}-x^{\star}\right\|+\frac{C}{L} \cdot \frac{1-\left(\frac{1-\gamma}{\kappa}\right)^{p+1}}{\frac{1-\gamma}{\kappa}}\right] \\
& \leq \kappa^{p+1}\left[\left\|x^{0}-x^{\star}\right\|+\frac{C \kappa}{L(\kappa+\gamma-1)}\right] .
\end{aligned}
$$

Hence, inequality 7 is proved.

From Lemma III.5, we know that the last missing piece is to show that the values $x_{i}^{k}$ and $\nabla f_{i}^{k}$ fall inside the quantization interval at every iteration $k$. The following assumption presents conditions on the number of bits $n$ and the initial quantization intervals $C_{\alpha}$ and $C_{\beta}$, which guarantee that for each iteration, $x_{i}^{k}$ and $\nabla f_{i}^{k}$ in Algorithm 2 fall inside the changing quantization intervals and the quantization errors decrease linearly with the constant $\kappa$, which further implies that Algorithm 2 converges to the global optimum linearly with the same rate $\kappa$.

Assumption III.6. We assume that the number of bits $n$ and the initial quantization intervals $C_{\alpha}$ and $C_{\beta}$ satisfy

$$
\begin{aligned}
& a_{1}+a_{2} \frac{C_{\alpha}}{2^{n+1}}+a_{3} \frac{C_{\beta}}{2^{n+1}} \leq \frac{C_{\alpha}}{2} \\
& b_{1}+b_{2} \frac{C_{\alpha}}{2^{n+1}}+b_{3} \frac{C_{\beta}}{2^{n+1}} \leq \frac{C_{\beta}}{2},
\end{aligned}
$$

with

$$
\begin{aligned}
a_{1} & =\frac{(\kappa+1)\left\|x^{0}-x^{\star}\right\|}{\kappa}, \quad a_{3}=\frac{M d \bar{m}(\kappa+1)}{L(\kappa+\gamma-1)} \\
a_{2} & =\frac{M d \bar{m} L_{\max } \kappa(\kappa+1)+M \bar{m} L(\kappa+\gamma-1)}{L \kappa(\kappa+\gamma-1)}, \\
b_{1} & =\frac{L_{\max }(\kappa+1)\left\|x^{0}-x^{\star}\right\|}{\kappa}, \\
b_{2} & =\frac{L_{\max } d \bar{m}(\kappa+1)\left(L_{\max } M \kappa+L \kappa+L \gamma-L\right)}{L \kappa(\kappa+\gamma-1)}, \\
b_{3} & =\frac{L_{\max } M d \bar{m} \kappa(\kappa+1)+L d \bar{m}(\kappa+\gamma-1)}{L \kappa(\kappa+\gamma-1)} .
\end{aligned}
$$

Remark III.7. Assumption III.6 can always be satisfied by increasing $n, C_{\alpha}$ and $C_{\beta}$.

Remark III.8. For a fixed n, inequalities (8) and (9) represent two polyhedral constraints on $C_{\alpha}$ and $C_{\beta}$. Therefore, the minimal $C_{\alpha}$ and $C_{\beta}$ can be computed by solving a simple $L P$ problem, i.e. minimizing $C_{\alpha}+C_{\beta}$ subject to $C_{\alpha} \geq 0$, $C_{\beta} \geq 0$, and inequalities (8) and (9). Since the minimal $n$ is 
the smallest value for which the LP problem has a feasible solution, the minimal $n$ can be found by testing feasibility of the LP problem.

Lemma III.9. If Assumption III.6 is satisfied, then for any $k \geq 0$ the values of $x_{i}^{k}$ and $\nabla f_{i}^{k}$ in Algorithm 2 fall inside of the quantization intervals of $Q_{\alpha, i}^{k}$ and $Q_{\beta, i}^{k}$, i.e. $\| x_{i}^{k}-$ $\bar{x}_{\alpha, i}^{k} \|_{\infty} \leq \frac{l_{\alpha, i}^{k}}{2}$ and $\left\|\nabla f_{i}^{k}-\bar{\nabla} f_{\beta, i}^{k}\right\|_{\infty} \leq \frac{l_{\beta, i}^{k}}{2}$.

Proof: We will prove Lemma III.9 by induction.

- Base case: At $k=0$, since $C_{\alpha}$ and $C_{\beta}$ are positive numbers and $\hat{x}_{i}^{-1}$ and $x_{i}^{0}$ are initialized to zero, it holds that $\left\|x_{i}^{0}-\bar{x}_{\alpha, i}^{0}\right\|_{\infty}=\left\|x_{i}^{0}-\hat{x}_{i}^{-1}\right\|_{\infty} \leq \frac{l_{\alpha, i}^{0}}{2}=\frac{C_{\alpha}}{2}$ and $\left\|\nabla f_{i}^{0}-\bar{\nabla} f_{\beta, i}^{0}\right\|_{\infty}=\left\|\nabla f_{i}\left(x_{\mathcal{N}_{i}}^{0}\right)-\hat{\nabla} f i\left(\hat{x}_{\mathcal{N}_{i}}^{-1}\right)\right\|_{\infty} \leq$ $\frac{l_{\beta, i}^{0}}{2}=\frac{C_{\beta}}{2}$.

- Induction step: Let $g \geq 0$ be given and suppose that $\left\|x_{i}^{k}-\bar{x}_{\alpha, i}^{k}\right\|_{\infty} \leq \frac{l_{\alpha, i}^{k}}{2}$ and $\left\|\nabla f_{i}^{k}-\bar{\nabla} f_{\beta, i}^{k}\right\|_{\infty} \leq \frac{l_{\beta, i}^{k}}{2}$ for $k \leq g$. We will prove that

$$
\left\|x_{i}^{g+1}-\bar{x}_{\alpha, i}^{g+1}\right\|_{\infty} \leq \frac{l_{\alpha, i}^{g+1}}{2}
$$

and

$$
\left\|\nabla f_{i}^{g+1}-\bar{\nabla} f_{\beta, i}^{g+1}\right\|_{\infty} \leq \frac{l_{\beta, i}^{g+1}}{2}
$$

for $i=1, \cdots, M$. 1. We first show (10). From Algorithm 2, we know

$$
\begin{aligned}
& \left\|x_{i}^{g+1}-\bar{x}_{\alpha, i}^{g+1}\right\|_{\infty}=\left\|x_{i}^{g+1}-\hat{x}_{i}^{g}\right\|_{\infty} \leq\left\|x^{g+1}-\hat{x}^{g}\right\|_{\infty} \\
& =\left\|x^{g+1}-x^{g}-\sum_{i=1}^{M} E_{i}^{T} \alpha_{i}^{g}\right\|_{\infty} \\
& \leq\left\|x^{g+1}-x^{g}\right\|_{\infty}+\left\|\sum_{i=1}^{M} E_{i}^{T} \alpha_{i}^{g}\right\|_{\infty} \\
& \leq\left\|x^{g+1}-x^{\star}\right\|_{\infty}+\left\|x^{g}-x^{\star}\right\|_{\infty}+\left\|\sum_{i=1}^{M} E_{i}^{T} \alpha_{i}^{g}\right\|_{\infty} \\
& \leq\left\|x^{g+1}-x^{\star}\right\|_{2}+\left\|x^{g}-x^{\star}\right\|_{2}+\sum_{i=1}^{M}\left\|\alpha_{i}^{g}\right\|_{2} .
\end{aligned}
$$

From Lemma III.5 and the assumption of the induction, it follows that the above expression is upper-bounded by

$$
\begin{aligned}
\leq & \kappa^{g+1}\left[\left\|x^{0}-x^{\star}\right\|+\frac{C \kappa}{L(\kappa+\gamma-1)}\right] \\
& +\kappa^{g}\left[\left\|x^{0}-x^{\star}\right\|+\frac{C \kappa}{L(\kappa+\gamma-1)}\right]+\frac{M \bar{m} C_{\alpha} \kappa^{g}}{2^{n+1}} .
\end{aligned}
$$

We substitute $C=\frac{M d \bar{m}\left(L_{\max } C_{\alpha}+C_{\beta}\right)}{2^{n+1}}$ and simplify it as

$$
=\kappa^{g+1}\left[a_{1}+a_{2} \cdot \frac{C_{\alpha}}{2^{n+1}}+a_{3} \cdot \frac{C_{\beta}}{2^{n+1}}\right],
$$

with the parameters defined as in Assumption III.6. Since inequality (8) holds, the term above is bounded by $\frac{C_{\alpha}}{2} \kappa^{g+1}$. Thus, inequality (10) holds. In the following, we prove that inequality (11) is true.

$$
\left\|\nabla f_{i}^{g+1}-\bar{\nabla} f_{\beta, i}^{g+1}\right\|_{\infty}=\left\|\nabla f_{i}^{g+1}-\hat{\nabla} f_{i}^{g}\right\|_{\infty}
$$

$$
\begin{aligned}
= & \left\|\nabla f_{i}\left(\hat{x}_{\mathcal{N}_{i}}^{g+1}\right)-\nabla f_{i}^{g}\left(\hat{x}_{\mathcal{N}_{i}}^{g}\right)+\beta_{i}^{g}\right\|_{\infty} \\
\leq & \left\|\nabla f_{i}\left(\hat{x}_{\mathcal{N}_{i}}^{g+1}\right)-\nabla f_{i}\left(\hat{x}_{\mathcal{N}_{i}}^{g}\right)\right\|_{\infty}+\left\|\beta_{i}^{g}\right\|_{\infty} \\
\leq & \| \nabla f_{i}\left(\hat{x}_{\mathcal{N}_{i}}^{g+1}\right)-\nabla f_{i}\left(\hat{x}_{\mathcal{N}_{i}}^{g}\left\|_{2}+\right\| \beta_{i}^{g} \|_{2}\right. \\
\leq & L_{i}\left\|\hat{x}_{\mathcal{N}_{i}}^{g+1}-\hat{x}_{\mathcal{N}_{i}}^{g}\right\|+\left\|\beta_{i}^{g}\right\| \\
\leq & L_{i}\left\|x_{\mathcal{N}_{i}}^{g+1}-x_{\mathcal{N}_{i}}^{g}\right\| \\
& +L_{i} \sum_{j \in \mathcal{N}_{i}}\left(\left\|\alpha_{j}^{g+1}\right\|+\left\|\alpha_{j}^{g}\right\|\right)+\left\|\beta_{i}^{g}\right\| \\
\leq & L_{i}\left\|x^{g+1}-x^{g}\right\|+L_{i} \sum_{j \in \mathcal{N}_{i}}\left(\left\|\alpha_{j}^{g+1}\right\|+\left\|\alpha_{j}^{g}\right\|\right)+\left\|\beta_{i}^{g}\right\| \\
\leq & L_{\max }\left(\left\|x^{g+1}-x^{\star}\right\|+\left\|x^{g}-x^{\star}\right\|\right) \\
& +L_{\max } \sum_{j \in \mathcal{N}_{i}}\left(\left\|\alpha_{j}^{g+1}\right\|+\left\|\alpha_{j}^{g}\right\|\right)+\left\|\beta_{i}^{g}\right\| .
\end{aligned}
$$

Again using Lemma III.5 and the assumption of the induction, it follows that the above is upper-bounded by

$$
\begin{aligned}
\leq & L_{\max } \kappa^{g+1}\left(\left\|x^{0}-x^{\star}\right\|+\frac{C \kappa}{L(\kappa+\gamma-1)}\right) \\
& +L_{\max } \kappa^{g}\left(\left\|x^{0}-x^{\star}\right\|+\frac{C \kappa}{L(\kappa+\gamma-1)}\right) \\
& +\frac{L_{\max } \bar{m} \sum_{j \in \mathcal{N}_{i}}\left(l_{\alpha, j}^{g+1}+l_{\alpha, j}^{g}\right)}{2^{n+1}}+\frac{d \bar{m} l_{\beta, i}^{g}}{2^{n+1}} \\
\leq & L_{\max } \kappa^{g+1}\left(\left\|x^{0}-x^{\star}\right\|+\frac{C \kappa}{L(\kappa+\gamma-1)}\right) \\
& +L_{\max } \kappa^{g}\left(\left\|x^{0}-x^{\star}\right\|+\frac{C \kappa}{L(\kappa+\gamma-1)}\right) \\
& +\frac{L_{\max } d \bar{m} C_{\alpha}\left(\kappa^{g+1}+\kappa^{g}\right)}{2^{n+1}}+\frac{d \bar{m} C_{\beta} \kappa^{g}}{2^{n+1}} .
\end{aligned}
$$

By substituting $C=\frac{M d \bar{m}\left(L_{\max } C_{\alpha}+C_{\beta}\right)}{2^{n+1}}$, we simplify the expression above as

$$
=\kappa^{g+1} \cdot\left[b_{1}+b_{2} \cdot \frac{C_{\alpha}}{2^{n+1}}+b_{3} \cdot \frac{C_{\beta}}{2^{n+1}}\right],
$$

with the parameters defined as in Assumption III.6. Since inequality (8) holds, the term above is bounded by $\frac{C_{\beta}}{2} \kappa^{g+1}=\frac{l_{\beta, i}^{g+1}}{2}$. Thus, inequality (11) holds.

We conclude that by the principle of induction, the values of $x_{i}^{k}$ and $\nabla f_{i}^{k}$ in Algorithm 2 fall inside of the quantization intervals of $Q_{\alpha, i}^{k}$ and $Q_{\beta, i}^{k}$, i.e. $\left\|x_{i}^{k}-\bar{x}_{\alpha, i}^{k}\right\|_{\infty} \leq \frac{l_{\alpha, i}^{k}}{2}$ and $\left\|\nabla f_{i}^{k}-\bar{\nabla} f_{\beta, i}^{k}\right\|_{\infty} \leq \frac{l_{\beta, i}^{k}}{2}$ for all $k \geq 0$.

After showing Lemma III.4, Lemma III.5 and Lemma III.9, we are ready to present the main theorem.

Theorem III.10. If Assumptions III.2, III.3 and III.6 hold, then for $k \geq 0$ the sequence $\left\{x^{k}\right\}$ generated by Algorithm 2 converges to the optimum linearly with the constant $\kappa$ and satisfies

$$
\left\|x^{p+1}-x^{\star}\right\| \leq \kappa^{p+1}\left[\left\|x^{0}-x^{\star}\right\|+\frac{C \kappa}{L(\kappa+\gamma-1)}\right] .
$$

with $C=\frac{M d \bar{m}\left(L_{\max } C_{\alpha}+C_{\beta}\right)}{2^{n+1}}$. 
Proof: Since Assumption III.2, III.3 and III.6 hold, Lemma III.9 shows that for each iteration the values $x_{i}^{k}$ and $\nabla f_{i}^{k}$ always fall inside of the quantization intervals of $Q_{\alpha, i}^{k}$ and $Q_{\beta, i}^{k}$. From Lemma III.5, we know that the computation error of the gradient $\left\|e^{k}\right\|$ decreases linearly with the constant $\kappa$ and $x^{k}$ satisfies inequality (12).

The complexity bound in Proposition II.3 states that if the error is equal to zero, the algorithm converges linearly with the constant $1-\gamma$. After imposing quantization on the algorithm, it still converges to the global optimum linearly but with a larger constant $\kappa>1-\gamma$. We conclude that with the proposed quantization design, the linear convergence of the algorithm is preserved, but the constant of the convergence rate is enlarged in order to compensate for the deficiencies from limited communication.

\section{NumericAl EXAMPLE}

This section illustrates the theoretical findings of the paper and demonstrates the performance of Algorithm 2 by solving a distributed least squares problem

$$
\min _{x \in \mathbb{R}^{n_{x}}} f(x)=\sum_{i=1}^{M} f_{i}\left(x_{\mathcal{N}_{i}}\right)=\sum_{i=1}^{M} x_{\mathcal{N}_{i}}^{T} H_{i} x_{\mathcal{N}_{i}}+h_{i} x_{\mathcal{N}_{i}} .
$$

We randomly generate a connected network with $M=20$ agents, and degree $d=8$. Each sub-system has two variables, i.e. $m_{i}=2$. Each agent has a local cost function $f_{i}\left(x_{\mathcal{N}_{i}}\right)=$ $x_{\mathcal{N}_{i}}^{T} H_{i} x_{\mathcal{N}_{i}}+h_{i} x_{\mathcal{N}_{i}}$, where $H_{i}=I$. The parameter $\gamma$ is equal to $\gamma=\frac{\sigma_{f}}{L}=\frac{2}{8}=0.25$ and $\kappa$ is set to be $\kappa=0.9 \geq$ $1-\gamma$. The parameters in Assumption III.6 are $a_{1}=10.5$, $a_{2}=551.1, a_{3}=506.6, b_{1}=10.5, b_{2}=524.4$ and $b_{3}=524.4$, respectively. The minimum number of bits is equal to $n_{\min }=11$. In the simulation of Fig. $1, n$ is set to 11, 13 and 15, and the initial quantization intervals $C_{\alpha}$ and $C_{\beta}$ are set to corresponding minimum values satisfying Assumption III.6. Fig. 1 shows the performance of Algorithm 2. We can observe that the proposed distributed algorithm with quantization converges to the global optimum linearly and the performance is improved when the number of bits $n$ is increased. The algorithm is implemented in Matlab, and the optimum $x^{\star}$ is computed by Matlab as well. Due to truncation and rounding errors, the accuracy of the exact solution, i.e. with zero error, is limited to a value around $10^{-15}$. Fig. 2 shows the relationship between the number of bits $n$ and the corresponding minimal initial quantization intervals $C_{\alpha}$ and $C_{\beta}$ satisfying Assumption III.6. As $n$ increases, the required minimal $C_{\alpha}$ and $C_{\beta}$ decrease.

\section{REFERENCES}

[1] A. Beck and M. Teboulle. A fast iterative shrinkage thresholding algorithm for linear inverse problems. SIAM Journal on Imaging Sciences, pages 183-202, 2009.

[2] R. Carli, F. Fagnani, P. Frasca, T. Taylor, and R. Zampieri. Average consensus on networks with transmission noise or quantization. In 9th European Control Conf., 2007.

[3] O. Devolder, F. Glineur, and Y. Nesterov. First-order methods of smooth convex optimization with inexact oracle. Mathematical Programming, 146:37-75, 2014.

[4] Q. T. Dinh, I. Necoara, and M. Diehl. Fast inexact decomposition algorithms for large-scale separable convex optimization. arXiv preprint arXiv:1212,4275, 2012.

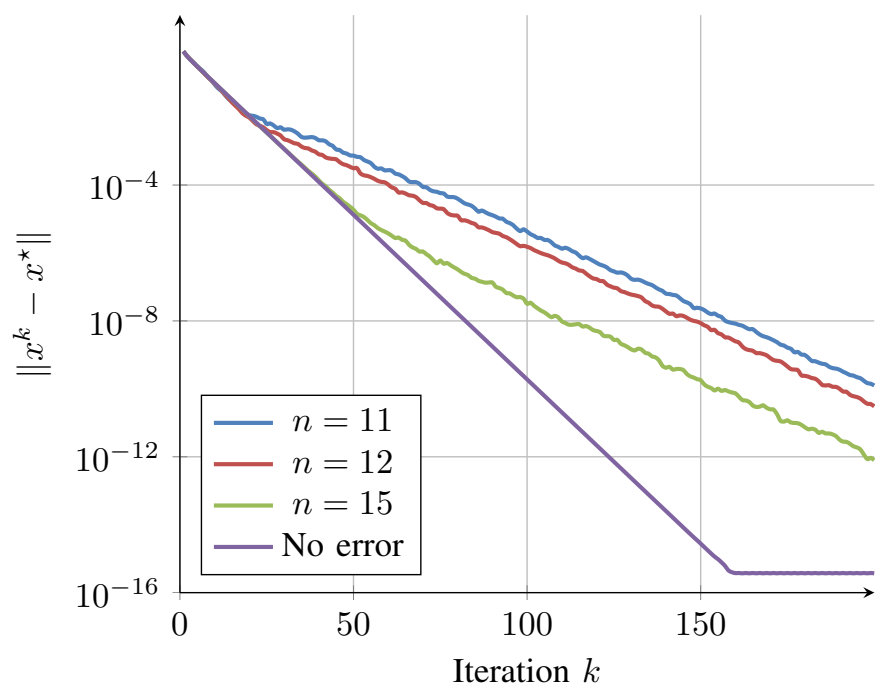

Fig. 1: Comparison of the performance of Algorithm 2 for different $n$ and corresponding minimum $C_{\alpha}$ and $C_{\beta}$, and the exact algorithm (no errors).

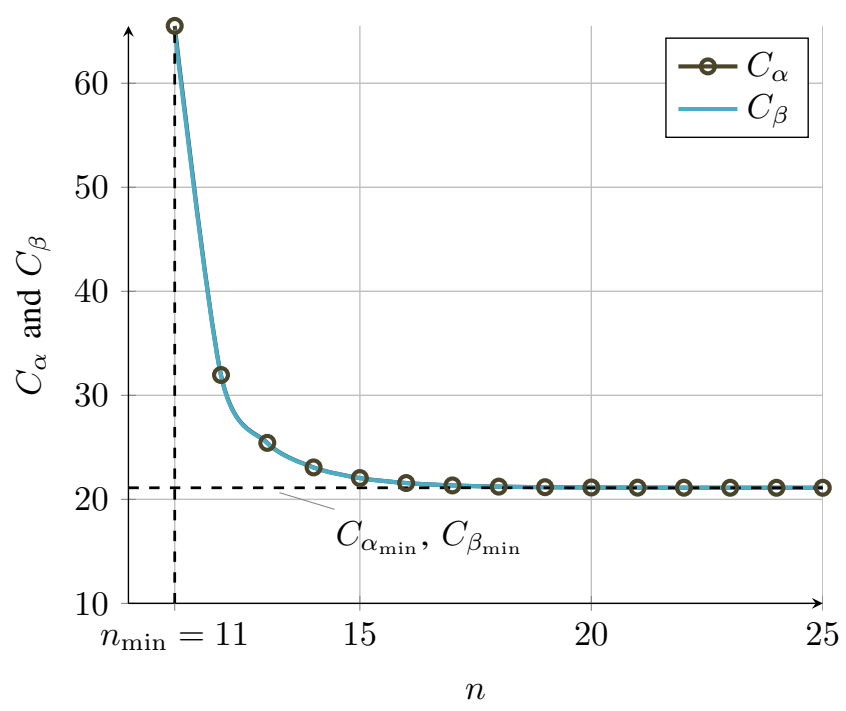

Fig. 2: Relationship between the number of bits $n$ and the minimum initial quantization intervals $C_{\alpha}$ and $C_{\beta}$ satisfying Assumption III.6.

[5] A. Kashyap, T. Basar, and R. Srikant. Quantized consensus. Automatica, 43:1192-1203, 2007.

[6] V. Nedelcu, I. Necoara, and I. Dumitrache. Complexity of an inexact augmented lagrangian method: Application to constrained MPC. In 19th World Congress of IFAC, 2014.

[7] A. Nedic, A. Olshevsky, A. Ozdaglar, and J.N. Tsitsiklis. Distributed subgradient methods and quantization effects. In 47th IEEE Conf. on Decision and Control, pages 4177-4184, 2008.

[8] Y. Pu, M.N. Zeilinger, and C.N. Jones. Inexact fast alternating minimization algorithm for distributed model predictive control. In 53rd IEEE Conf. on Decision and Control, pages 5915-5921, 2014.

[9] M. Schmidt, N. L. Roux, and F. Bach. Convergence rates of inexact proximal-gradient methods for convex optimization. In 25th Annual Conf. on Neural Information Processing Systems, pages 6819-6824, 2011.

[10] D. Thanou, E. Kokiopoulou, Y. Pu, and P. Frossard. Distributed average consensus with quantization refinement. IEEE Transactions on Signal Processing, 61:194-205, 2013. 